\title{
From the stellar properties of HD 219134 to the internal compositions of its transiting exoplanets
}

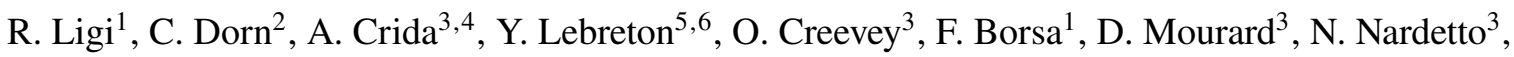 \\ I. Tallon-Bosc ${ }^{7}$, F. Morand ${ }^{3}$, and E. Poretti ${ }^{1}$
}

${ }^{1}$ INAF - Osservatorio Astronomico di Brera, Via E. Bianchi 46, 23807 Merate, Italy e-mail: roxanne.ligi@inaf.it

2 Institut of Computational Sciences, University of Zurich, Winterthurerstrasse 190, 8057 Zurich, Switzerland

${ }^{3}$ Université Côte d'Azur, Observatoire de la Côte d'Azur, CNRS, Laboratoire Lagrange, Bd de l'Observatoire, CS 34229, 06304 Nice Cedex 4, France

${ }^{4}$ Institut Universitaire de France, 103 boulevard Saint-Michel, 75005 Paris, France

${ }^{5}$ LESIA, Observatoire de Paris, PSL Research University, CNRS, Sorbonne Universités, UPMC Université Paris 06, Université Paris Diderot, Sorbonne Paris Cité, 92195 Meudon Cedex, France

${ }^{6}$ Univ. Rennes, CNRS, IPR (Institut de Physique de Rennes) - UMR 6251, 35000 Rennes, France

7 Univ. Lyon, Univ. Lyon1, Ens de Lyon, CNRS, Centre de Recherche Astrophysique de Lyon UMR 5574, 69230 Saint-Genis-Laval, France

Received 5 July 2019 / Accepted 20 September 2019

\begin{abstract}
Context. The harvest of exoplanet discoveries has opened the area of exoplanet characterisation. But this cannot be achieved without a careful analysis of the host star parameters.

Aims. The system of HD 219134 hosts two transiting exoplanets and at least two additional non-transiting exoplanets. We revisit the properties of this system using direct measurements of the stellar parameters to investigate the composition of the two transiting exoplanets.

Methods. We used the VEGA/CHARA interferometer to measure the angular diameter of HD 219134. We also derived the stellar density from the transits light curves, which finally gives a direct estimate of the mass. This allowed us to infer the mass, radius, and density of the two transiting exoplanets of the system. We then used an inference model to obtain the internal parameters of these two transiting exoplanets.

Results. We measure a stellar radius, density, and mass of $R_{\star}=0.726 \pm 0.014 R_{\odot}, \rho_{\star}=1.82 \pm 0.19 \rho_{\odot}$, and $M_{\star}=0.696 \pm 0.078 M_{\odot}$, respectively; there is a correlation of 0.46 between $R_{\star}$ and $M_{\star}$. This new mass is lower than that derived from the C2kSMO stellar evolutionary model, which provides a mass range of $0.755-0.810( \pm 0.040) M_{\odot}$. Moreover, we find that planet $b$ and $c$ have smaller radii than previously estimated of $1.500 \pm 0.057$ and $1.415 \pm 0.049 R_{\oplus}$ respectively; this clearly puts these planets out of the gap in the exoplanetary radii distribution and validates their super-Earth nature. Planet $b$ is more massive than planet $c$, but the former is possibly less dense. We investigate whether this could be caused by partial melting of the mantle and find that tidal heating due to non-zero eccentricity of planet $b$ may be powerful enough.

Conclusions. The system of HD 219134 constitutes a very valuable benchmark for both stellar physics and exoplanetary science. The characterisation of the stellar hosts, and in particular the direct determination of the stellar density, radius, and mass, should be more extensively applied to provide accurate exoplanets properties and calibrate stellar models.
\end{abstract}

Key words. stars: fundamental parameters - stars: individual: HD 219134 - planetary systems - techniques: interferometric methods: numerical - planets and satellites: fundamental parameters

\section{Introduction}

The huge harvest of exoplanets discovered by the space telescopes Kepler (Borucki et al. 2010) and CoRoT (Baglin 2003) has led to the understanding that exoplanets are the rule rather than the exception. We have now moved to the era of exoplanet characterisation, and the next challenge is to understand how common rocky planets are and if any are suitable for life. The most interesting exoplanets to study are certainly the transiting exoplanets, as the transit light curve allows us to know the planetary radius. An additional radial velocity (RV) follow-up provides the planetary mass and thus the planetary density. The three ingredients to estimate planetary bulk composition are then gathered. But this is only true if the stellar radius and mass are known. Up to now, most of transiting exoplanet hosts have been very faint, driven by the search for exoplanets rather than their characterisation, often leading to inaccurate and/or imprecise stellar parameters. This makes the characterisation of the whole exoplanetary system difficult and the determination of the exoplanetary internal structure approximate.

Several methods can be employed to obtain the stellar parameters. Concerning the mass, it is often determined indirectly, as only stars in binary systems can have their mass directly measured if the system inclination is known. However, if an exoplanet is transiting its host star, the density of the star can be directly inferred from the transit light curve (Seager \& MallénOrnelas 2003). Then, in the case of bright stars, the radius can be directly determined using interferometry, which is a high angular 
resolution technique aimed at measuring the angular diameter of stars with a precision up to a few percent (Baines et al. 2010; Boyajian et al. 2012a,b; Huber et al. 2012; Creevey et al. 2012, 2015; Ligi et al. 2012, 2016, e.g.). The mass can thus be directly computed from the transit and interferometric measurements. This method has recently been used by Crida et al. (2018a,b) to derive the mass of the very bright star $55 \mathrm{Cnc}$ with a precision of $6.6 \%$ using the interferometric diameter measured by Ligi et al. (2016) and the density from the transit light curve obtained for 55 Cnc $e$ (Bourrier et al. 2018). This yielded the best characterisation of the transiting super-Earth $55 \mathrm{Cnc} e$ so far and a new estimate of its internal composition.

HD 219134 (HIP 114622, GJ 892) is also a bright $(V=5.57)$ K3V star 6.5 parsecs away from us. Motalebi et al. (2015) first detected four exoplanets around the star from RV measurements using the High Accuracy Radial velocity Planet Searcher for the Northern hemisphere (HARPS-N) on the Telescopio Nazionale Galileo (TNG). Moreover, Spitzer time-series photometric observations allowed the detection of the transit of planet $b$, leading to the estimate of a rocky composition. The same year, Vogt et al. (2015) claimed the detection of six planets around HD 219134 from the analysis of RV obtained with the HIgh Resolution Echelle Spectrometer (HIRES) on Keck I Observatory and the Levy Spectrograph at the Automated Planet Finder Telescope (Lick Observatory). These authors derived similar periods for planets $b, c$, and $d$, the other diverging because of the different Keplerian analysis of the RV signal leading to a different number of planets. Later, Gillon et al. (2017) reported additional Spitzer observations of the system that led to the discovery of the transit of the second innermost planet, HD $219134 c$. The two innermost planets seem rocky, but more interestingly, planet $c$ shows a higher density while it has a lower mass than planet $b$. The detailed planetary data and their relative differences place additional constraints on their interiors with implications to their formation and evolution.

In this paper, we report new observations of HD 219134 using the Visible spEctroGraph and polArimeter (VEGA) instrument on the Center for High Angular Resolution Astronomy (CHARA) interferometric array that led to a new accurate determination of angular diameter of this star (Sect. 2). In Sect. 3, we determine the stellar radius and density, and derive the joint probability density function (PDF) of the stellar mass and radius independently of stellar models. We then use the PDF to compute the new parameters of the two transiting exoplanets and we revisit those of the non-transiting exoplanets in Sect. 4.1. Finally, we derive the internal composition of planets $b$ and $c$ in Sect. 4.2 using a planetary interior model, and we discuss the possible cause of the different densities of planets $b$ and $c$ in Sect. 4.3. We conclude in Sect. 5.

\section{Interferometric measurement of the angular diameter with VEGA/CHARA}

We used the technique of interferometry to measure the angular diameter of HD 219134. These measurements constitute the first step to determine the other fundamental parameters of this star.

\subsection{Observations and data reduction}

We observed HD 219134 from 2016 to 2018 using the VEGA/CHARA instrument at visible wavelengths (see Table 1) and medium resolution. The spectro-interferometer VEGA (Mourard et al. 2009; Ligi et al. 2013) is based on the CHARA array (ten Brummelaar et al. 2005), which takes advantage of the six $1 \mathrm{~m}$ telescopes distributed in a Y-shape to insure wide $(u, v)$ coverage. It can be used at medium (5000) or high spectral resolution (30000) and with baselines ranging from 34 to $331 \mathrm{~m}$ in the two telescope (2T), 3T, or 4T modes. The observations were calibrated following the sequence calibrator - science star calibrator, and were performed using different configurations (Table 1), mainly in the 2T mode at once to optimise the signalto-noise ratio $(\mathrm{S} / \mathrm{N})$ of the observations. The calibrator stars were selected into the SearchCal software ${ }^{1}$ (Table 2), and we used the uniform disc diameter in the $R$ band (UDDR) found in the JSDC2 (Bourgés et al. 2014) or SearchCal (Chelli et al. 2016) catalogue otherwise. However, for conservative reasons, we decided to use an uncertainty of $7 \%$ or that given in the JSDC1 (Bonneau et al. 2006) if higher. We selected the calibrators with several criteria: in the neighbourhood of the star, discarding variable stars and multiple systems, and with high squared visibilities, allowing an optimal measurement of the instrumental transfer function. Finally, the data were reduced using the vegadrs pipeline (Mourard et al. 2009, 2011) developed at Observatoire de la Côte d'Azur. For each observation, we selected two nonredundant spectral bands of $20 \mathrm{~nm}$ wide centred at 685,705 , or $725 \mathrm{~nm}$ in most cases to derive the squared visibility $\left(V^{2}\right)$, but the reddest band is sometimes of bad quality or features absorption lines and cannot be used. In total, we collected 36 data points, which are shown in Fig. 1.

\subsection{Angular diameter}

The squared visibilities that we obtained (Fig. 1, coloured filled circles) are well spread on the $V^{2}$ curve. We note some dispersion around $0.7 \times 10^{8} / \mathrm{rad}$ (corresponding to the E1E2 configuration) but it is taken into account in the computation of the error on the angular diameter. We also adopted a conservative approach by setting a minimum error of $5 \%$ on $V^{2}$ to balance the known possible bias with VEGA (Mourard et al. 2012, 2015).

We used the LITpro software (Tallon-Bosc et al. 2008) to fit our visibility points and derive the angular diameter of HD 219134 and its related uncertainty. Taking a model of uniform disc, we obtained $\theta_{\mathrm{UD}}=0.980 \pm 0.020$ millisecond of arc (mas; Table 3). However, this simple representation is not realistic and we thus used a linear limb-darkening (LD) model to refine it, as the LD diameter $\left(\theta_{\mathrm{LD}}\right)$ cannot be directly measured. We must indeed use empirical tables of LD coefficients $\mu_{\lambda}$, which depend on the effective temperature $T_{\text {eff }}$, gravity $\log (g)$, and metallicity $[\mathrm{Fe} / \mathrm{H}]$ at a given wavelength $\lambda$. We used Claret \& Bloemen (2011) tables as a start in the $R$ and $I$ band since we observed between 685 and $720 \mathrm{~nm}$, and proceeded on interpolations to obtain a reliable LD coefficient at our wavelength, as described in Ligi et al. (2016). The LD coefficients in Claret \& Bloemen (2011) tables are given in steps of $250 \mathrm{~K}$ for $T_{\text {eff }}$, 0.5 dex for $\log (g)$ and less uniform steps for $[\mathrm{Fe} / \mathrm{H}]$. We set a starting value of these parameters to perform our interpolation in between the surrounding values. We searched in the literature previous values of $\log (g)$ and $[\mathrm{Fe} / \mathrm{H}]$ through the SIMBAD database $^{2}$ and calculated the median and standard deviation of the values given there (see selected values in Table A.1 and the medians in Table 3). Beforehand, we eliminated aberrant values and values obtained before the year 2000, to insure recent and probably more reliable estimates. Since many values of the metallicity could be derived from a same data set, and because the uncertainty in the various papers can be higher than our standard deviation $(0.05 \mathrm{dex})$, we set the uncertainty on $[\mathrm{Fe} / \mathrm{H}]$

\footnotetext{
http://www.jmmc.fr/searchcal_page.htm

2 Available at http://cdsweb.u-strasbg.fr/
} 
Table 1. Observing log.

\begin{tabular}{|c|c|c|c|c|c|c|c|c|c|c|}
\hline MJD & $\begin{array}{l}\text { Date } \\
\text { UT }\end{array}$ & Telescopes & $\begin{array}{c}\text { Bas. length } \\
{[\mathrm{m}]}\end{array}$ & Seq. & $S / N$ & $V^{2}$ & $\sigma V_{\text {Stat }}^{2}$ & $\sigma V_{\text {Syst }}^{2}$ & $\begin{array}{c}\lambda \\
{[\mathrm{nm}]}\end{array}$ & $\begin{array}{c}\delta \lambda \\
{[\mathrm{nm}]}\end{array}$ \\
\hline 57621.451 & $2016-08-21$ & W2E2 & 105.30 & $\mathrm{C} 1-\mathrm{sci}-\mathrm{C} 1$ & 4.1 & 0.206 & 0.050 & $1.43 \mathrm{E}-03$ & 685 & 20 \\
\hline 57711.177 & 2016-11-19 & E1E2 & 65.79 & C1-sci-C1 & 13.9 & 0.694 & 0.050 & $1.78 \mathrm{E}-03$ & 705 & 20 \\
\hline 57711.177 & & & 65.79 & & 15.0 & 0.751 & 0.050 & $1.76 \mathrm{E}-03$ & 725 & 20 \\
\hline 57711.269 & 2016-11-19 & E1E2 & 65.48 & $\mathrm{C} 1-\mathrm{sci}-\mathrm{C} 1$ & 10.9 & 0.546 & 0.050 & $1.41 \mathrm{E}-03$ & 705 & 20 \\
\hline 57711.292 & 2016-11-19 & E1E2 & 65.36 & $\mathrm{C} 1-\mathrm{sci}-\mathrm{C} 1$ & 11.3 & 0.567 & 0.050 & $1.69 \mathrm{E}-03$ & 705 & 20 \\
\hline 57712.150 & $2016-11-20$ & $\mathrm{~W} 1 \mathrm{~W} 2$ & 107.82 & $\mathrm{C} 1-\mathrm{sci}-\mathrm{C} 2$ & 5.6 & 0.280 & 0.050 & $1.48 \mathrm{E}-03$ & 705 & 20 \\
\hline 57712.172 & 2016-11-20 & W1W2 & 107.20 & C2-sci-C1 & 6.0 & 0.300 & 0.050 & $1.64 \mathrm{E}-03$ & 705 & 20 \\
\hline 57712.172 & & & 107.20 & & 2.2 & 0.111 & 0.050 & $9.24 \mathrm{E}-04$ & 725 & 20 \\
\hline 57712.221 & 2016-11-20 & W1W2 & 103.69 & $\mathrm{C} 1-\mathrm{sci}-\mathrm{C} 2$ & 3.8 & 0.189 & 0.050 & $9.39 \mathrm{E}-04$ & 705 & 20 \\
\hline 57712.221 & & & 103.69 & & 6.1 & 0.304 & 0.050 & $1.46 \mathrm{E}-03$ & 725 & 20 \\
\hline 57712.245 & 2016-11-20 & W1W2 & 101.20 & C2-sci-C1 & 4.9 & 0.245 & 0.050 & $1.16 \mathrm{E}-03$ & 705 & 20 \\
\hline 57712.245 & & & 101.20 & & 5.3 & 0.267 & 0.050 & $1.21 \mathrm{E}-03$ & 725 & 20 \\
\hline 57734.160 & 2016-12-12 & $\mathrm{S} 1 \mathrm{~S} 2$ & 29.52 & C1-sci-C1 & 11.6 & 0.987 & 0.085 & $5.80 \mathrm{E}-04$ & 705 & 20 \\
\hline 57734.160 & & & 29.52 & & 11.6 & 0.958 & 0.083 & $5.38 \mathrm{E}-04$ & 725 & 20 \\
\hline 57960.258 & $2017-07-26$ & W1W2 & 95.26 & C3-sci-C3 & 5.9 & 0.296 & 0.050 & $1.63 \mathrm{E}-03$ & 705 & 20 \\
\hline 57960.258 & & & 95.26 & & 5.8 & 0.289 & 0.050 & $1.51 \mathrm{E}-03$ & 725 & 20 \\
\hline 57960.279 & $2017-07-26$ & W1W2 & 96.32 & C3-sci-C4 & 6.1 & 0.304 & 0.050 & $2.14 \mathrm{E}-03$ & 705 & 20 \\
\hline 57960.279 & & & 96.32 & & 6.7 & 0.334 & 0.050 & $2.28 \mathrm{E}-03$ & 725 & 20 \\
\hline 57960.302 & $2017-07-26$ & W1W2 & 97.91 & C4-sci-C4 & 6.6 & 0.331 & 0.050 & $2.57 \mathrm{E}-03$ & 705 & 20 \\
\hline 57960.302 & & & 97.91 & & 7.5 & 0.373 & 0.050 & $2.74 \mathrm{E}-03$ & 725 & 20 \\
\hline 57960.324 & $2017-07-26$ & W1W2 & 99.79 & C4-sci-C3 & 5.8 & 0.290 & 0.050 & $2.07 \mathrm{E}-03$ & 705 & 20 \\
\hline 57960.324 & & & 99.79 & & 5.9 & 0.294 & 0.050 & $2.02 \mathrm{E}-03$ & 725 & 20 \\
\hline 57964.369 & 2017-07-30 & E1E2 & 61.12 & C4-sci-C3 & 9.8 & 0.489 & 0.050 & $1.06 \mathrm{E}-03$ & 705 & 20 \\
\hline 57964.369 & & & 61.12 & & 9.1 & 0.455 & 0.050 & $9.33 \mathrm{E}-04$ & 725 & 20 \\
\hline 57964.510 & $2017-07-30$ & E1E2 & 65.87 & C3-sci-C3 & 15.0 & 0.750 & 0.050 & $2.25 \mathrm{E}-03$ & 705 & 20 \\
\hline 57964.510 & & & 65.87 & & 16.5 & 0.823 & 0.050 & $2.18 \mathrm{E}-03$ & 725 & 20 \\
\hline 57965.343 & 2017-07-31 & E1E2 & 59.00 & C3-sci-C3 & 14.7 & 0.737 & 0.050 & 2.19E-03 & 705 & 20 \\
\hline 57965.343 & & & 59.00 & & 14.9 & 0.745 & 0.050 & $1.79 \mathrm{E}-03$ & 725 & 20 \\
\hline 58299.481 & 2018-06-30 & E1E2 & 63.21 & C3-sci-C3 & 10.5 & 0.525 & 0.050 & $1.24 \mathrm{E}-03$ & 703 & 20 \\
\hline 58299.481 & & & 63.21 & & 11.5 & 0.577 & 0.050 & $1.29 \mathrm{E}-03$ & 723 & 20 \\
\hline 58299.501 & 2018-06-30 & E1E2 & 64.25 & C3-sci-C3 & 13.2 & 0.659 & 0.050 & $1.58 \mathrm{E}-03$ & 703 & 20 \\
\hline 58299.501 & & & 64.25 & & 13.3 & 0.665 & 0.050 & $1.50 \mathrm{E}-03$ & 723 & 20 \\
\hline 58302.334 & 2018-07-03 & W1W2 & 95.87 & C3-sci-C4 & 7.6 & 0.378 & 0.050 & $2.77 \mathrm{E}-03$ & 703 & 20 \\
\hline 58302.334 & & & 95.87 & & 6.3 & 0.361 & 0.057 & $2.21 \mathrm{E}-03$ & 723 & 20 \\
\hline 58302.351 & 2018-07-03 & W1W2 & 96.91 & C4-sci-C3 & 3.7 & 0.220 & 0.060 & $1.47 \mathrm{E}-03$ & 703 & 20 \\
\hline 58302.351 & & & 96.91 & & 7.7 & 0.385 & 0.050 & $2.41 \mathrm{E}-03$ & 723 & 20 \\
\hline
\end{tabular}

Notes. From left to right, the table shows the observing date, telescopes used, projected baseline lengths, observing sequence ("sci" refers to the science target, and "C-" to the calibrator; see Table 2), the S/N, measured squared visibility, statistical and systematic errors, observation wavelength, and corresponding bandwidth.

Table 2. Angular diameters of the calibrators used.

\begin{tabular}{llcc}
\hline \hline Cal. & Name & $\theta_{\text {UD }} \pm \sigma \theta_{\text {UD }}[\mathrm{mas}]$ & Ref. \\
\hline C1 & HD 1279 & $0.183 \pm 0.013$ & $(1)$ \\
C2 & HD 209419 & $0.158 \pm 0.011$ & $(1)$ \\
C3 & HD 218376 & $0.188 \pm 0.013$ & $(2)$ \\
C4 & HD 205139 & $0.174 \pm 0.017$ & $(2)$ \\
\hline
\end{tabular}

References. (1) JSDC2 (Bourgés et al. 2014); (2) SearchCal (Chelli et al. 2016).

to 0.1 dex. Concerning the starting $T_{\text {eff }}$ value, we used that fitted through the spectral energy distribution $\left(\mathrm{SED} ; T_{\mathrm{eff}, \mathrm{SED}}=4839 \mathrm{~K}\right.$, Sect. 3.2). Since the star is close by (distance, $d=6.533 \pm 0.038$ pc, Table 3), we set the reddening to $A_{\mathrm{v}}=0.0 \pm 0.01 \mathrm{mag}$. This value is consistent with the extinction given by the Stilism
(Lallement et al. 2014) 3D map of the galactic interstellar matter $(E(B-V)=0 \pm 0.014)$ but corresponds to a smaller uncertainty on the extinction (0.0034 mag).

For each filter, we first computed the linear interpolation of the LD coefficients corresponding to the surrounding values of $[\mathrm{Fe} / \mathrm{H}], \log (g)$ and $T_{\text {eff }}$ of our star. We then averaged the two coefficients coming out from each filter to get a final coefficient. Then, we used the LITpro software to fit our data using a linear LD model while fixing in the model our new LD coefficient. This results in $\theta_{\mathrm{LD}}=1.035 \pm 0.021$ mas ( $2 \%$ precision). It has to be noted that using different LD laws does not significantly change the final diameter as we are not sensitive to it in the first lobe of visibility. If we set $T_{\text {eff }}=4750 \mathrm{~K}, \log (g)=4.5 \mathrm{dex}$, and $[\mathrm{Fe} / \mathrm{H}]=0.1$ dex, a quadratic LD law described by Claret \& Bloemen (2011) yields $\theta_{\mathrm{LD}}=1.047 \pm 0.022 \mathrm{mas}$ in the $R$ band (using the LD coefficients $a_{1, R}=0.5850$ and $b_{2, R}=0.1393$ given in the table) and $\theta_{\mathrm{LD}}=1.033 \pm 0.022$ mas in the $I$ band (taking 


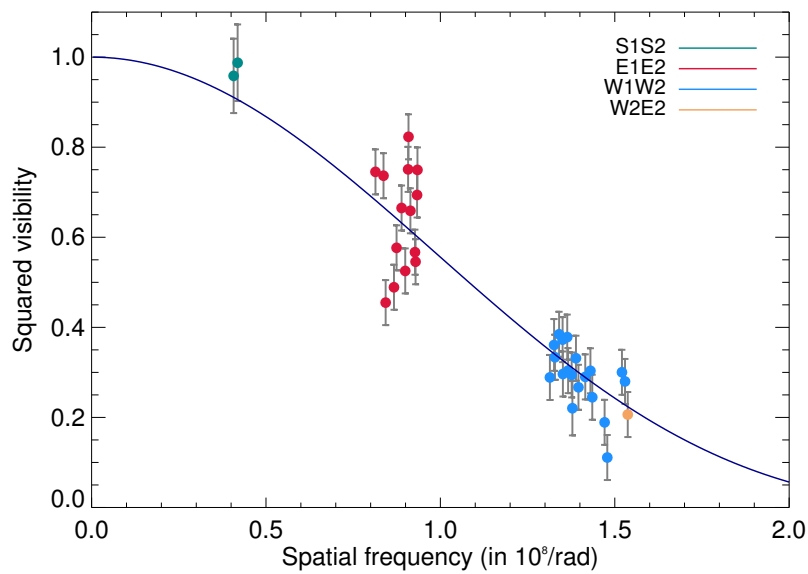

Fig. 1. Squared visibilities obtained with VEGA/CHARA for HD 219134. The different colours represent the data points obtained with different baselines. The solid line represents the model of LD diameter.

$a_{1, I}=0.4490$ and $\left.b_{2, I}=0.1828\right)$. Similarly, averaging $a_{1, R}$ and $a_{1, I}$ coefficients on the one hand, and $b_{2, R}$ and $b_{2, I}$ on the other hand, leads to $\theta_{\mathrm{LD}}=1.040 \pm 0.022 \mathrm{mas}$, and thus a value within the error bars of our first estimate. Our determined angular diameter is smaller than that previously measured with the CHARA Classic beam combiner (1.106 \pm 0.007 mas; Boyajian et al. 2012b). Although their visibilities seem more precise, we stress that we obtain higher spatial frequency data, which resolves the star better. The angular diameter derived from the SED $\theta_{\text {SED }}$ is also very consistent with our measurement (1.04 mas, see Sect. 3.2).

\section{Stellar parameters}

The new angular diameter constitutes the basis of our analysis. It is now possible to determine the other stellar parameters from our interferometric measurements, and to compare these parameters with those derived from stellar evolution models.

\subsection{Radius, density, and mass}

The stellar radius is generally derived using the distance and angular diameter as follows: $\theta_{\mathrm{LD}}=2 R_{\star} / d$. As for the mass, Crida et al. (2018a,b) showed the importance of using the correlation between the stellar mass and radius to reduce the possible solutions in the mass-radius plane. We took the same approach to derive $R_{\star}$ and $M_{\star}$. The PDF of $R_{\star}$, called $f_{R_{\star}}$, can be expressed as a function of the PDF of the observables $\theta_{\mathrm{LD}}$ (angular diameter) and $\pi$ (parallax), called $f_{\theta}$ and $f_{\pi}$ respectively. This gives

$f_{R_{\star}}(R)=\frac{R_{0}}{R^{2}} \int_{0}^{\infty} t f_{\pi}\left(\frac{R_{0} t}{R}\right) f_{\theta}(t) \mathrm{d} t$,

where $R_{0}$ is a constant (see Crida et al. 2018a, for the proof). Concerning Gaia parallaxes, Stassun \& Torres (2018) have reported that an offset of $-82 \pm 33 \mu$ as is observed, while Lindegren et al. (2018) have provided $-30 \mu a s$. In any case, these offsets are within the uncertainty of the parallax for HD 219134 and do not impact significantly our results. As advised by Luri et al. (2018), we only used the parallax and its error given in the Gaia DR2 catalogue (Gaia Collaboration 2016, 2018), keeping in mind the possible offsets and that for such bright stars, there might still be unknown offsets that DR3 and DR4 will provide.

We found $R_{\star}=0.726 \pm 0.014 \rho_{\odot}$, which is a lower value than that found by Boyajian et al. $\left(2012 \mathrm{~b}, R_{\star}=0.778 \pm 0.005 R_{\odot}\right)$. Our
Table 3. Stellar parameters of HD 219134.

\begin{tabular}{|c|c|c|}
\hline Parameter & Value & Ref. \\
\hline \multicolumn{3}{|c|}{ Coordinates and photometry } \\
\hline RA (J2000) & $23^{\mathrm{h}} 13^{\mathrm{m}} 16^{\mathrm{s}} .97$ & Gaia DR2 (1) \\
\hline $\operatorname{Dec}(\mathrm{J} 2000)$ & $+57^{\circ} 10^{\prime} 06^{\prime \prime} .08$ & Gaia DR2 (1) \\
\hline$\pi[\mathrm{mas}]$ & $153.081 \pm 0.0895$ & Gaia DR2 $^{(1)}$ \\
\hline$d[\mathrm{pc}]$ & $6.533 \pm 0.038$ & Gaia DR2 $^{(1),(a)}$ \\
\hline $\mathrm{V}[\mathrm{mag}]$ & $5.570 \pm 0.009$ & $\operatorname{CDS}^{(2)}$ \\
\hline $\mathrm{K}$ [mag] & $3.25 \pm 0.01$ & $\operatorname{CDS}^{(2)}$ \\
\hline$L_{\star}\left[L_{\odot}\right]$ & 0.30 & Gaia DR2 ${ }^{(1)}$ \\
\hline \multicolumn{3}{|c|}{ Interferometric parameters } \\
\hline$\theta_{\mathrm{UD}}[\mathrm{mas}]$ & $0.980 \pm 0.020(2 \%)$ & This work, Sect. 2.2 \\
\hline$u_{\lambda}$ & 0.588 & This work ${ }^{(b)}$, Sect. 2.2 \\
\hline$\theta_{\mathrm{LD}}[\mathrm{mas}]$ & $1.035 \pm 0.021(2 \%)$ & This work, Sect. 2.2 \\
\hline \multicolumn{3}{|c|}{ Fixed parameters } \\
\hline $\mathrm{A}_{\mathrm{v}}[\mathrm{mag}]$ & $0.0 \pm 0.01$ & This work, Sect. 3.2 \\
\hline$[\mathrm{Fe} / \mathrm{H}]$ & $0.07 \pm 0.1$ & SIMBAD $^{(c)}$, Sect. 2.2 \\
\hline $\log (g)\left[\mathrm{cm} \mathrm{s}^{2}\right]$ & $4.57 \pm 0.14$ & SIMBAD $^{(c)}$, Sect. 2.2 \\
\hline \multicolumn{3}{|c|}{ Fitted parameters } \\
\hline$T_{\text {eff, SED }}[K]$ & $4839 \pm 25$ & This work, Sect. 3.2 \\
\hline$\theta_{\mathrm{SED}}[\mathrm{mas}]$ & $1.043 \pm 0.013$ & This work, Sect. 3.2 \\
\hline$F_{\text {bol }}\left[\operatorname{erg~s~cm}{ }^{-2} \times 10^{8}\right]$ & $19.86 \pm 0.21$ & This work, Sect. 3.2 \\
\hline \multicolumn{3}{|c|}{ Measured and computed parameters } \\
\hline$T_{\text {eff }}[K]$ & $4858 \pm 50$ & This work, Sect. 3.2 \\
\hline$R_{\star}\left[R_{\odot}\right]$ & $0.726 \pm 0.014$ & This work, Sect. 3.1 \\
\hline$L_{\star}\left[L_{\odot}\right]$ & $0.264 \pm 0.004$ & This work, Sect. 3.2 \\
\hline$\rho_{\star}\left[\rho_{\odot}\right]$ & $1.82 \pm 0.19$ & This work, Sect. 3.1 \\
\hline$M_{\star}\left[M_{\odot}\right]$ & $0.696 \pm 0.078$ & This work ${ }^{(d)}$, Sect. 3.1 \\
\hline$M_{\text {grav }, \star}\left[M_{\odot}\right]$ & $0.72 \pm 0.23$ & This work ${ }^{(e)}$, Sect. 3.1 \\
\hline $\operatorname{Corr}\left(R_{\star}, M_{\star}\right)$ & 0.46 & This work, Sect. 3.1 \\
\hline \multicolumn{3}{|c|}{ Stellar model inferences with C2kSMO } \\
\hline$R_{\star}\left[R_{\odot}\right]$ & $0.727 \pm 0.017$ & This work, Sect. 3.3 \\
\hline$M_{\star}\left[M_{\odot}\right]$ & $0.755 \pm 0.040$ & This work, Sect. 3.3 \\
\hline$\rho_{\star}\left[\rho_{\odot}\right]$ & $1.96 \pm 0.22$ & This work, Sect. 3.3 \\
\hline $\mathrm{Age}_{\star}[\mathrm{Gyr}]$ & 9.3 & This work, Sect. 3.3 \\
\hline
\end{tabular}

Notes. ${ }^{(a)}$ From $\pi .{ }^{(b)}$ Computed from Claret \& Bloemen (2011) tables. ${ }^{(c)}$ Averaged from the values available in the SIMBAD database (Wenger et al. 2000); see text for details. ${ }^{(d)}$ From $\rho_{\star}$ and $R_{\star} ;{ }^{(e)}$ from $\log (g)$.

References. ${ }^{(1)}$ Gaia Collaboration (2018); ${ }^{(2)}$ Oja (1993).

uncertainty on $R_{\star}$ is clearly dominated by the uncertainty on the angular diameter because we took the parallax from Gaia DR2, which is very precise $(0.06 \%)$.

The stellar density $\rho_{\star}$ can be derived from the transit duration, period and depth (Seager \& Mallén-Ornelas 2003). In our system, we have two transiting exoplanets. We computed the stellar density independently for both transits using the data given by Gillon et al. (2017) and found $1.74 \pm 0.22$ and $2.04 \pm 0.37 \rho_{\odot}$ for planets $b$ and $c$, respectively. We note that the density coming from the analysis of the light curve for HD $219134 c$ is less precise than that of HD $219134 \mathrm{~b}$. This comes from the transit light curves themselves, which are more complete and more precise for planet $b$. Combining both densities, we obtained $1.82 \pm 0.19 \rho_{\odot}$, which we use in the rest of our analysis. We computed the uncertainty following a classical propagation of 


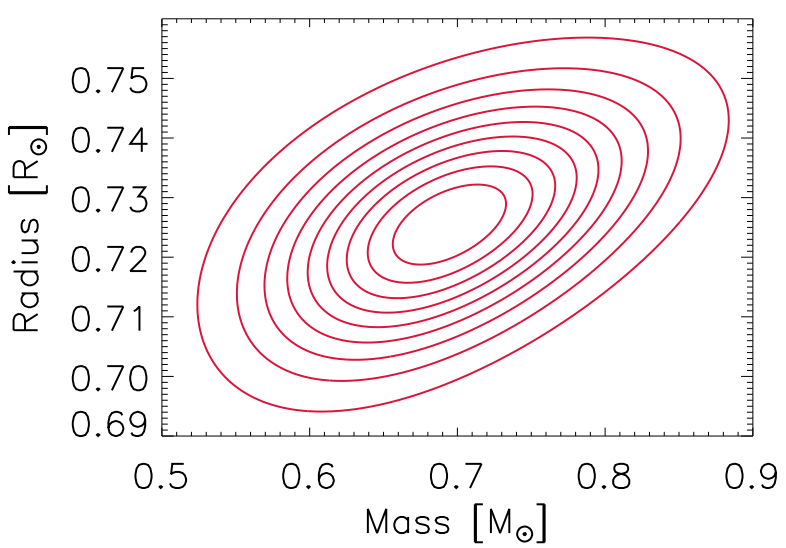

Fig. 2. Joint likelihood of the radius and mass of the star HD 219134. The 9 plain red contour lines separate 10 equal-sized intervals between 0 and the maximum of Eq. (2).

errors and found a value close but different, and with a bigger error bar compared to that given in Gillon et al. (2017). The joint likelihood of $M_{\star}$ and $R_{\star}$ can be expressed as

$\mathcal{L}_{M R \star}(M, R)=\frac{3}{4 \pi R^{3}} \times f_{R_{\star}}(R) \times f_{\rho_{\star}}\left(\frac{3 M}{4 \pi R^{3}}\right)$,

as described in Crida et al. (2018a) and where $f_{\rho_{\star}}$ is the PDF of the stellar density (Fig. 2). The calculated correlation coefficient between $R_{\star}$ and $M_{\star}$ is 0.46 . Our computation yields $M_{\star}=0.696 \pm 0.078 M_{\odot}$, which is consistent with the value determined directly from $\log (g)$ and $R_{\star}$ but with a better precision. For reference, other authors derived $0.763 \pm 0.076 M_{\odot}$ (Boyajian et al. 2012b) using the relation by Henry \& McCarthy (1993), and $0.81 \pm 0.03 M_{\odot}$ (Gillon et al. 2017) using stellar evolution modelling. In this latter case, the uncertainty corresponds to the internal source of error of the model and is thus underestimated.

\subsection{Bolometric flux, effective temperature, and luminosity}

To derive the $T_{\text {eff }}$ of the star we combined the angular diameter with its bolometric flux $F_{\text {bol }}$ using

$T_{\mathrm{eff}}=\left(\frac{4 \times F_{\mathrm{bol}}}{\sigma_{\mathrm{SB}} \theta_{\mathrm{LD}}^{2}}\right)^{0.25}$,

where $\sigma_{\mathrm{SB}}$ is the Stefan-Boltzmann constant. This implies computation of the bolometric flux, which we derived from the stellar photometry as described in the next subsection.

\subsubsection{Bolometric flux}

We determine the bolometric flux $F_{\text {bol }}$ and its uncertainty in the following way. We retrieved photometric data from the literature made available by the VizieR Photometry tool ${ }^{3}$. These photometry converted-to-flux measurements were fitted to the BaSeL empirical library of spectra (Lejeune et al. 1997), using a non-linear least-squared minimisation algorithm (LevenbergMarquardt). The spectra are characterised by $T_{\text {eff }},[\mathrm{M} / \mathrm{H}]$, and $\log (g)$. To convert these spectra to observed spectra they need to be scaled by $\left(R_{\star} / d\right)$ and reddened for interstellar extinction $A_{\mathrm{v}}$. Thus, each model spectrum is characterised by these five parameters. In practice most of these parameters are degenerate,

3 http://vizier.u-strasbg.fr/vizier/sed/

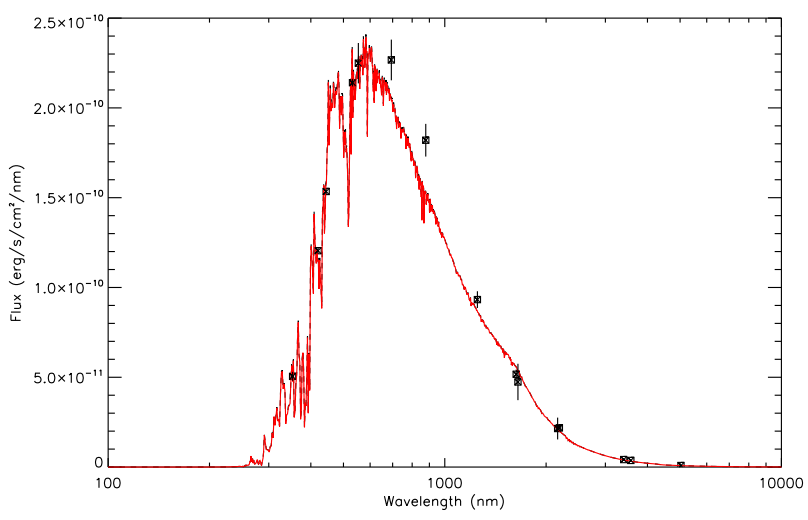

Fig. 3. Photometric data (black squares) and fitted model (solid red line) from the BaSeL library of spectra.

so it is necessary to fix a subset of these. For each minimisation performed we fixed [M/H], $\log (g)$, and $A_{\mathrm{v}}$ i.e. we only fitted $T_{\text {eff }}$ and $(R / d \propto \theta)$, and then we integrated under the resulting scaled and unreddened empirical spectrum to obtain $F_{\text {bol }}$.

To properly estimate the uncertainties in the parameters we repeated this method 1000 times to obtain a distribution of $F_{\text {bol }}$. Each of these minimisations had different fixed values of $[\mathrm{M} / \mathrm{H}], \log (g)$, and $A_{\mathrm{v}}$ obtained by drawing random numbers from gaussian distributions characterised by the following: $[\mathrm{M} / \mathrm{H}]=+0.07 \pm 0.10, \log (g)=4.57 \pm 0.14$, and $A_{\mathrm{v}}=0.00 \pm 0.01 \mathrm{mag}$, as discussed in Sect. 2.2. The initial values of $T_{\text {eff }}$ and $(R / d)$ were obtained by drawing them from a random uniform distribution with values between 4100 and $5700 \mathrm{~K}$ and between 4.0 and 8.0. Using the resulting distribution of $F_{\mathrm{bol}}$, we calculated $F_{\text {bol }}=19.86 \pm 0.21 \mathrm{erg} \mathrm{s} \mathrm{cm}^{-2} \times 10^{8}$. In the same way, we also estimated the $T_{\text {eff }}$ from the resulting distributions of the best-fitted $T_{\text {eff }}\left(T_{\text {eff,SED }}\right)$ and the angular diameter $(R / d)$ converted to units of mas $\left(\theta_{\mathrm{SED}}\right)$, although these latter two are not used any further in this work.

The best-fit model spectrum is shown in Fig. 3 in red, along with photometric data points in black. Overall, the model fits the data well, except for two points that are above the fit, but removing the two outliers did not change the results. These over-fluxes could come from a close star or another undetermined source, although we could not verify these hypotheses.

\subsubsection{Effective temperature and luminosity}

We derived the effective temperature $T_{\text {eff }}$ from $F_{\text {bol }}$ and $\theta_{\mathrm{LD}}$ using Eq. (3) to obtain $4858 \pm 50 \mathrm{~K}$. This is in very good agreement with the $T_{\text {eff }}$ determined by Gaia $\left(4787_{-73}^{+92} \mathrm{~K}\right)$ and with that determined through the SED fitting $(4839 \pm 25 \mathrm{~K})$, which has a lower uncertainty. We finally obtained the luminosity using the distance and $F_{\text {bol }}$ as follows:

$L_{\star}=4 \pi d^{2} F_{\text {bol }}$.

The errors on these final parameters were estimated following a classical propagation of errors (see Ligi et al. 2016, for details). The Gaia luminosity is $L_{\star}=0.30 L_{\odot}$, which is in good agreement with our value $\left(L_{\star}=0.264 \pm 0.004 L_{\odot}\right)$ considering the documented possible systematic errors. All final stellar parameters are reported in Table 3.

\subsection{Comparison with stellar evolution models}

HD 219134 is now a well-characterised star thanks to our direct measurements of its radius and density, providing in turn its 
mass. Therefore, it constitutes a good benchmark to be compared to stellar evolution models.

We thus confront our measurements (mass, radius, and density) to the values that can be inferred from stellar evolution modelling. For that purpose, we have used the $\mathrm{C}_{2} \mathrm{kSMO}^{4}$ stellar model optimisation pipeline (Lebreton \& Goupil 2014) to find the mass, age, and initial metallicity of the stellar model that best fits the luminosity, effective temperature, and surface metallicity (hereafter observational constraints) of HD 219134 given in Table 3. The procedure operates via a Levenberg-Marquardt minimisation performed on stellar models calculated on-the-fly with the Cesam2k (Morel \& Lebreton 2008) stellar evolution code $(\mathrm{C} 2 \mathrm{kSMO}$ is described in detail in Lebreton \& Goupil 2014).

For a given set of input parameters and physics of a stellar model (nuclear reaction rates, equation of state, opacities, atmospheric boundary conditions, convection formalism, and related mixing-length parameter for convection, element diffusion and mixing, solar mixture of heavy elements, initial helium content, etc.), we can therefore infer the mass, age, and initial metallicity of the best model for HD 219134 with internal error bars resulting from the uncertainties on the observational constraints. However, among these inputs, many are still very uncertain or even unknown. Accordingly, to get a reasonable estimate of the accuracy of the results, we performed several model optimisations, each of which correspond to a different set of input physics and parameters. We varied the following - most uncertain inputs:

Solar mixture. We investigated the effects of using either the GN93 (Grevesse \& Noels 1993) or the AGSS09 (Asplund et al. 2009) mixture. However, we point out that, although still widely used, the GN93 mixture is no longer valid. The AGSS09 mixture is based on carefully updated atomic data and on a 3D time-dependent hydrodynamic model of the solar atmosphere, while the GN93 mixture was inferred through a 1D model of the solar atmosphere. As discussed by, for example, Nordlund et al. (2009), the 3D model reproduces the observations of the solar atmosphere remarkably well, while the $1 \mathrm{D}$ model atmosphere does not. The AGSS09 mixture should therefore be preferred.

Convection description. We used either the classical mixing-length theory (usually referred to as MLT; Böhm-Vitense 1958) or the Canuto, Goldmann, and Mazzitelli formalism (usually referred to as CGM; Canuto et al. 1996).

External boundary conditions. We investigated the effects of using either the approximate Eddington's grey radiative $T-\tau$ law ( $T$ is the temperature, $\tau$ the optical depth) or the more physical $T-\tau$ law extracted from Model Atmospheres in Radiative and Convective Scheme (MARCS) model atmospheres (Gustafsson et al. 2008). Although MARCS models are classical 1D model atmospheres in local thermodynamical equilibrium, they do include convection in the MLT formalism and use up-todate atomic and molecular data (see e.g. Gustafsson et al. 2008). Therefore, these models represent an important progress with respect to the grey law and should be preferred.

Initial helium abundance. This quantity is not accessible through the analysis of stellar spectra because helium lines are not formed in the spectra of cool and tepid stars. It is a major source of uncertainty in stellar model calculation. In stellar models the initial helium abundance is generally estimated from the

4 C2kSMO stands for "Cesam2k Stellar Model Optimisation."
$\Delta Y / \Delta Z$ galactic enrichment law ${ }^{5}$ to overcome this difficulty. Two different $\Delta Y / \Delta Z$ values are usually used: the value obtained from solar model calibration ${ }^{6}$ (chosen for instance in the new Bag of Stellar Tracks and Isochrones (BaSTI) stellar model grids; see Hidalgo et al. 2018), which is $\approx 1$ or the so-called galactic value, $\Delta Y / \Delta Z \approx 2$ (Casagrande et al. 2007) adopted for instance in the Modules for Experiments in Stellar Astrophysics (MESA) grids by Coelho et al. (2015). The former depends on the input physics of the solar model while the latter is very uncertain (see e.g. Gennaro et al. 2010). On the other hand, the initial helium content can be estimated by modelling stars with available asteroseismic observational constraints. This is the case of 66 stars in the Kepler Legacy sample for which we obtained values of $\Delta Y / \Delta Z$ in the range $1-3$ with a mean of $(\Delta Y / \Delta Z)_{\text {seism }} \approx 2.3$ with the C2kSMO pipeline (see e.g. Silva Aguirre et al. 2017). Since no strong justification of what would be the best choice can be given, we investigated the impact of using the two values $\Delta Y / \Delta Z=1$ and 2 because the latter is also close to the mean Kepler Legacy asteroseismic value $(\Delta Y / \Delta Z)_{\text {seism }}$, but keeping in mind this remains the main source of uncertainty in our results.

More details on the uncertainties of stellar model inputs and their consequences can be found in Lebreton et al. (2014). To avoid such sources of uncertainties, direct measurements of stellar parameters should be preferred when possible.

Depending on the stellar model input physics and parameters, we obtained a large range of possible ages, between $\approx 0.2$ and 9.3 Gyr with large error bars. The range of possible masses is between 0.755 and $0.810 M_{\odot}$. The internal error bar on the inferred mass for an optimised stellar model based on a given set of inputs physics and parameters due to the uncertainty on the observational constraints (luminosity, effective temperature, and metallicity) is $\approx \pm 0.04 M_{\odot}$. This error bar appears to be small. Indeed, in the Levenberg-Marquardt minimisation the error bars on the free parameters are obtained as the diagonal coefficients of the inverse of the Hessian matrix and have been shown to be smaller than those provided with other minimisation techniques (see e.g. Silva Aguirre et al. 2017). The inferred stellar radii are in the range $0.727-0.728 R_{\odot}$ with an internal error bar of $\pm 0.017 R_{\odot}$, while the mean densities are in the range 1.96-2.09 $( \pm 0.22) \rho_{\odot}$. We chose as reference model for the star that based on the most appropriate input physics as explained in the description above (AGSS09 solar mixture and boundary conditions from MARCS model atmospheres), and the galactic value $\Delta Y / \Delta Z=2$ derived by Casagrande et al. (2007), which is also rather close to the Kepler Legacy seismic mean value $(\Delta Y / \Delta Z)_{\text {seism. }}$ This particular model has $M_{\star}=0.755 \pm 0.040 M_{\odot}$ and an age of $9.3 \mathrm{Gyr}$. Although this mass estimate is higher than the mass we derived from interferometry and transit by $\sim 8 \%$, the interval of solutions is consistent with our uncertainties. Similarly, our radius and density are consistent with those derived from the model $\left(0.727 \pm 0.017 R_{\odot}, 1.96 \pm 0.22 \rho_{\odot}\right.$, respectively). We point out that pushing the $\Delta Y / \Delta Z$ value from 2 to 3 would induce a change of mass from 0.755 to $0.719 M_{\odot}$, i.e. closer to the interferometric measure, but with a change in age from 9.3 to $13.8 \mathrm{Gyr}$, i.e. the age of the Universe; in our opinion this

$5 \Delta Y / \Delta Z=\left(Y-Y_{\mathrm{P}}\right) / Z$, where $Y_{P}$ is the primordial helium abundance in mass fraction, and $Y$ and $Z$ are the current helium and metallicity mass fractions, respectively.

6 In the solar model calibration process, the evolution of a $1 M_{\odot}$ model is calculated up to the known solar age. Its initial helium content and mixing-length parameter are fixed by the constraint that at solar age, the model has reached the observed values of the solar radius, luminosity, and surface metallicity. 
indicates that $\Delta Y / \Delta Z$ values that are too high are not realistic for this star.

We point out that, as is well-known in particular in the case of low-mass stars, the ages of stars are very poorly estimated when only the H-R diagram parameters and metallicity are known because of degeneracies in the stellar models (see e.g. Lebreton et al. 2014; Ligi et al. 2016). Furthermore, other values of the classical stellar parameters of HD 219134 have been reported in the literature. To see how these reported values can modify our results we optimised stellar models on the basis of the Folsom et al. (2018) results on $T_{\text {eff }}$ and $[\mathrm{Fe} / \mathrm{H}]$ and on $L_{\star}$ inferred from the SIMBAD HIPPARCOS $V$-magnitude. We obtained a similar range of masses $0.76-0.79 M_{\odot}$, while the models systematically point towards higher ages $10.2-13.8 \mathrm{Gyr}$, which is mainly due to the smaller $T_{\text {eff }}(4756 \pm 86 \mathrm{~K})$ derived by Folsom et al. (2018). It is also worth pointing out that, as noted by Johnson et al. (2016), the very high ages inferred from stellar models commonly found in the literature for HD 219134 seem to be in conflict with ages from activity which, although not very precise, span the range $\approx 3-9 \mathrm{Gyr}^{7}$.

\section{Planetary parameters and composition of the transiting exoplanets}

The precise and accurate stellar parameters that we have determined allow us to infer the parameters of the transiting exoplanets of the system. It is then possible to derive their internal composition using an inference scheme, and to verify if they stand in a dynamical point of view.

\subsection{Radius, density, and mass of the two transiting exoplanets}

The two planets HD $219134 b$ and $c$ transit their host star, and we can thus derive their properties. We computed the planetary radius $R_{\mathrm{p}}$ and mass $M_{\mathrm{p}}$ of each planet starting from the PDF of the stellar mass and radius. As explained by Crida et al. (2018a) concerning $55 \mathrm{Cnc} e$, for any $M_{\mathrm{p}}$ and $M_{\star}$, we can derive the associated semi-amplitude of the RV signal $K$ following Kepler's law, and for any pair of $R_{\mathrm{p}}$ and $R_{\star}$, we can derive the associated transit depth $\Delta F$. We took the $\Delta F, K$, and the period $P$ from Gillon et al. (2017) to calculate the PDF of the planetary mass and radius following the formula (see Sect. 3.1 of Crida et al. 2018a, for more details) :

$$
\begin{aligned}
f_{\mathrm{p}}\left(M_{\mathrm{p}}, R_{\mathrm{p}}\right) \propto & \iint \exp \left(-\frac{1}{2}\left(\frac{K\left(M_{\mathrm{p}}, M_{\star}\right)-K}{\sigma_{K}}\right)^{2}\right) \\
& \times \exp \left(-\frac{1}{2}\left(\frac{\Delta F\left(M_{\mathrm{p}}, M_{\star}\right)-\Delta F}{\sigma_{\Delta F}}\right)^{2}\right) \\
& \times \mathcal{L}_{M R \star}\left(M_{\star}, R_{\star}\right) \mathrm{d} M_{\star} \mathrm{d} R_{\star} .
\end{aligned}
$$

From this joint PDF, we compute the densities of both transiting exoplanets taking into account the correlation between $R_{\mathrm{p}}$ and $M_{\mathrm{p}}$ (Fig. 4).

The new values of the planetary parameters are given in Table 4. The radii of planets $b$ and $c$ are $1.500 \pm 0.057$ and $1.415 \pm 0.049 R_{\oplus}$, respectively. Because we find that the star is smaller than initially thought, the two planets appear

\footnotetext{
7 We estimated this age range from the empirical relation relating the CaII $\mathrm{H} \& \mathrm{~K}$ emission index $R_{\mathrm{HK}}^{\prime}$ and age derived by Mamajek \& Hillenbrand (2008), with the value of $R_{\mathrm{HK}}^{\prime}$ measured by Boro Saikia et al. (2018).
}

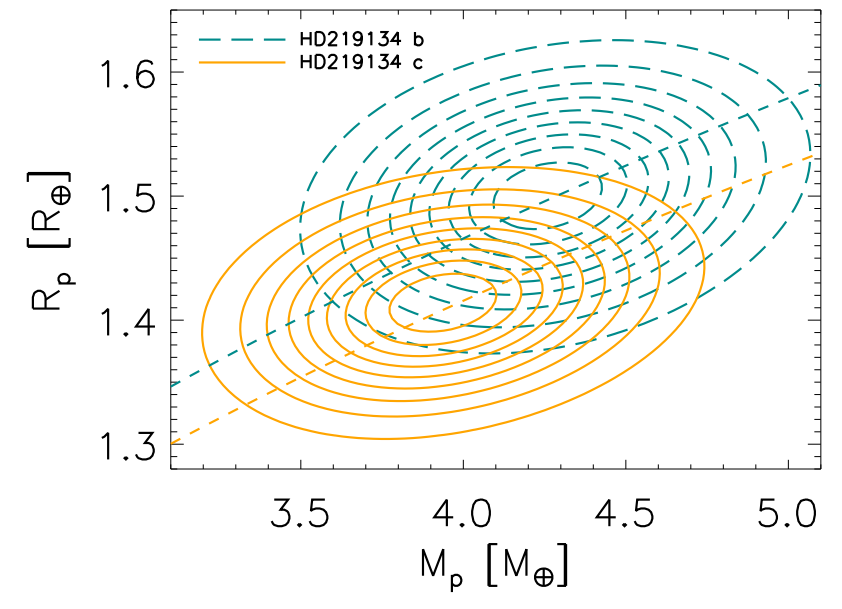

Fig. 4. Joint likelihood of the planetary mass and radius for planet $b$ (green long-dashed line) and planet $c$ (yellow solid line). The 9 contour lines separate 10 equal-sized intervals between 0 and the maximum of $f_{\mathrm{p}}\left(M_{\mathrm{p}}, R_{\mathrm{p}}\right)$. The dashed lines show the iso-densities corresponding to the mean densities of planets $b$ and $c$.

smaller as well; Gillon et al. (2017) give $R_{\mathrm{p}}=1.602 \pm 0.055$ and $1.511 \pm 0.047 R_{\oplus}$, and $M_{\mathrm{p}}=4.74 \pm 0.19$ and $4.36 \pm 0.22 M_{\oplus}$, for planets $b$ and $c$, respectively. This enforces the idea that the two planets lie in the super-Earth part of the distribution of exoplanetary radii set by Fulton et al. (2017).

Even more interestingly, planet $c$ presents a higher density than planet $b$, whereas it has smaller mass and radius. From the values in Table 4 , we get $\rho_{b} / \rho_{c}=0.901 \pm 0.157$ assuming $\rho_{b}$ and $\rho_{c}$ to be independent variables. But $\rho_{b}$ and $\rho_{c}$ are slightly correlated as they both depend on the stellar parameters. Estimating directly the ratio, the stellar parameters simplify out to

$\frac{\rho_{b}}{\rho_{c}}=\frac{M_{b} / R_{b}^{3}}{M_{c} / R_{c}^{3}}=\left(\frac{P_{b}}{P_{c}}\right)^{1 / 3}\left(\frac{\Delta F_{c}}{\Delta F_{b}}\right)^{3 / 2}\left(\frac{K_{b}}{K_{c}}\right)=0.905 \pm 0.131$,

where $P_{b}$ and $P_{c}$ are the orbital periods of the planets; we used a standard propagation of error. This is a larger difference than between the Earth and Venus (whose density is $0.944 \rho_{\oplus}$ ). A better knowledge of the transit depth would help discriminate between the density ratio and unity. We investigated the causes of this potential disparity in the next section.

We also updated the values of the minimum masses of planets $f$ and $d$, which as expected we find lower than previous estimates, and of their semi-major axes (Table 4) using Gillon et al. (2017) orbital solutions, as these planets are confirmed by several independent detection. Finally, we determined the habitable zone (HZ) of the star to verify if any of the exoplanets of this system lie in this zone. To compute the HZ, we used the method described by Jones et al. (2006), who adopted a conservative approach of this range of distances. We first computed the critical flux which depends on the $T_{\text {eff }}$ of the star, and we derived the inner and outer boundaries of the HZ (see Eqs. (1a)-(2b) of Jones et al. 2006, for details). As a result, we find that the HZ spreads from 0.46 to 0.91 au from the star and that no planet in the system is located in this area.

\subsection{Internal compositions}

The new mass and radius estimates allowed us to investigate the planetary interiors. Interestingly, there is a $10 \%$ density difference between the two planets (see Eq. (6)), which are otherwise 
Table 4. Parameters of the innermost exoplanets of the system HD 219134.

\begin{tabular}{lcccc}
\hline \hline Param. & HD 219134 $b$ & HD 219134c & HD 219134f & HD 219134d \\
\hline$R_{\mathrm{p}}\left[R_{\oplus}\right]$ & $1.500 \pm 0.057$ & $1.415 \pm 0.049$ & - & - \\
$M_{\mathrm{p}}\left[M_{\oplus}\right]$ & $4.27 \pm 0.34$ & $3.96 \pm 0.34$ & 6.60 & 14.64 \\
$\operatorname{Corr}\left(R_{\mathrm{p}}, M_{\mathrm{p}}\right)$ & 0.22 & 0.23 & - & - \\
$\rho_{\mathrm{p}}\left[\rho_{\oplus}\right]$ & $1.27 \pm 0.16$ & $1.41 \pm 0.17$ & - & - \\
$a[\mathrm{au}]$ & 0.037 & 0.062 & 0.139 & 0.225 \\
\hline
\end{tabular}

Notes. For the two transiting exoplanets, the mass is given, while for the two other planets the minimum planetary mass $M_{\mathrm{p}} \sin (i)$ is given.

Table 5. Median stellar abundances of HD 219134 from Hypatia catalog (Hinkel et al. 2014) after the outliers and duplicate studies were removed.

\begin{tabular}{ll}
\hline \hline Parameter & HD 219134 \\
\hline$[\mathrm{Fe} / \mathrm{H}]$ & $0.13 \pm 0.08$ \\
{$[\mathrm{Mg} / \mathrm{H}]$} & $0.16 \pm 0.14$ \\
{$[\mathrm{Si} / \mathrm{H}]$} & $0.17 \pm 0.15$ \\
{$[\mathrm{Na} / \mathrm{H}]$} & $0.22 \pm 0.08$ \\
{$[\mathrm{Al} / \mathrm{H}]$} & $0.26 \pm 0.07$ \\
{$[\mathrm{Ca} / \mathrm{H}]$} & $0.13 \pm 0.13$ \\
\hline
\end{tabular}

Notes. The unit is dex.

very similar in mass. Although the uncertainty in the density ratio allows for no interior difference between the planets, it is worth investigating what could cause this possible difference, which is larger than that between the Earth and Venus with a probability of about $70 \%$ (or stated differently, 30\% chance for a difference of less than 5\%). In fact, Eq. (6) suggests that there is a $50 \%$ chance that planet $b$ is more than $10 \%$ less dense than planet $c$.

The lower density of planet $b$ can be associated with secondary atmospheres or a rock composition that is enriched in very refractory elements (Dorn et al. 2018; Dorn \& Heng 2018). Recently, Bower et al. (2019) demonstrated that fully or partially molten mantle material can lower the bulk density of super-Earth up to $13 \%$. Therefore, a difference between the planetary densities may also be due to different melt fractions in both planets. In Sect. 4.2, we investigate this additional scenario and discuss its implications.

We start by solving an inference problem, for which we use the data of mass, radius (Sect. 4.1), stellar irradiation, and stellar abundances (Table 5) to infer the possible structures and compositions of both planets. Stellar abundances of rock-forming elements (e.g. $\mathrm{Fe}, \mathrm{Mg}, \mathrm{Si}$ ) are used as proxies for the rocky interiors to reduce interior degeneracy as proposed by Dorn et al. (2015). The differences between both planet interiors may provide evidence of their different formation or evolution history.

\subsubsection{Inference scheme}

We used the inference scheme of Dorn et al. (2017), which calculates possible interiors and their confidence ranges. Our assumptions for the interior model are similar to those in Dorn et al. (2017) and are summarised in the following. Since these two planets are smaller than $\sim 1.8 R_{\oplus}$, which is suggested to be the boundary between super-Earths and mini-Neptunes (Fulton et al. 2017), we consider that the planets are made of iron-rich cores, silicate mantles, and terrestrial-type atmospheres. In addition to following Dorn et al. (2017), we also allowed for some reduction of the mantle density as caused by a high melt fraction.

The interior parameters comprise:

- core size $r_{\text {core }}$;

- size of rocky interior $r_{\text {core+mantle; }}$;

- mantle composition (i.e. $\mathrm{Fe} / \mathrm{Si}_{\text {mantle }}, \mathrm{Mg} / \mathrm{Si}_{\text {mantle }}$ );

- reduction factor of mantle density $f_{\text {mantle }}$;

- pressure imposed by gas envelope $P_{\text {env }}$;

- temperature of gas envelope parametrised by $\alpha$ (see Eq. (10));

- mean molecular weight of gas envelope $\mu$.

The prior distributions of the interior parameters used in this study are stated in Table 6.

Our interior model uses a self-consistent thermodynamic model for solid state interiors from Dorn et al. (2017). For any given set of interior parameters, this model allows us to calculate the respective mass, radius, and bulk abundances and to compare them to the actual observed data. The thermodynamic model comprises the equation of state $(\mathrm{EoS})$ of pure iron by Bouchet et al. (2013) and of the light alloy FeSi by Hakim et al. (2018), assuming 2.5\% of FeSi similar to Earth's core. For the silicate-mantle, we used the model by Connolly (2009) to compute equilibrium mineralogy and density profiles given the database of Stixrude \& Lithgow-Bertelloni (2011). We allowed for a reduction of mantle densities as caused by the presence of melt. Unfortunately, the knowledge of EoS of melts is limited for pressures that occur in super-Earths (e.g. Spaulding et al. 2012; Bolis et al. 2016; Wolf \& Bower 2018). Therefore, we decided to use a very simplified approach in that we used a fudge factor $f_{\text {mantle }}$ that reduces the mantle density $\rho_{\text {mantle }}$ in each grid layer $i$ by $\rho_{\text {mantle }, i} \times\left(1-f_{\text {mantle }}\right)$.

For the gas layer, we used a simplified atmospheric model for a thin, isothermal atmosphere in hydrostatic equilibrium and ideal gas behaviour, which is calculated using the scale-height model (model II in Dorn et al. 2017). The model parameters that parametrise the gas layer and that we aim to constrain are the pressure at the bottom of the gas layer $P_{\text {env }}$, the mean molecular weight $\mu$, and the mean temperature (parametrised by $\alpha$, see below). The thickness of the opaque gas layer $d_{\text {env }}$ is given by

$d_{\mathrm{env}}=H \ln \frac{P_{\mathrm{env}}}{P_{\mathrm{out}}}$,

where the amount of opaque scale heights $H$ is determined by the ratio of $P_{\text {env }}$ and $P_{\text {out }}$. The quantity $P_{\text {out }}$ is the pressure level at the optical photosphere for a transit geometry that we fix to 20 mbar (Fortney et al. 2007). We allowed a maximum pressure $P_{\text {env }}$ equivalent to a Venus-like atmosphere (i.e. 100 bar). The scale height $H$ is expressed by

$H=\frac{T_{\text {env }} R^{*}}{g_{\text {env }} \mu}$, 
Table 6. Prior ranges for interior parameters.

\begin{tabular}{lll}
\hline \hline Parameter & Prior range & Distribution \\
\hline Core radius $r_{\text {core }}$ & $(0.01-1) r_{\text {core+mantle }}$ & Uniform in $r_{\text {core }}^{3}$ \\
$\mathrm{Fe} / \mathrm{Si}_{\text {mantle }}$ & $0-\mathrm{Fe} / \mathrm{Si}_{\text {star }}$ & Uniform \\
$\mathrm{Mg} / \mathrm{Si}_{\text {mantle }}$ & $\mathrm{Mg} / \mathrm{Si}_{\text {star }}$ & Gaussian \\
$f_{\text {mantle }}$ & $0 .-0.2$ & Uniform \\
Size of rocky interior $r_{\text {core+mantle }}$ & $(0.01-1) R_{\mathrm{p}}$ & Uniform in $r_{\text {core+mantle }}^{3}$ \\
Pressure imposed by gas envelope $P_{\text {env }}$ & $20 \mathrm{mbar}-100 \mathrm{bar}$ & Uniform in log-scale \\
Temperature of gas envelope $\alpha$ & $0.5-1$ & Uniform \\
Mean molecular weight of gas envelope $\mu$ & $16-50 \mathrm{~g} \mathrm{~mol}^{-1}$ & Uniform \\
\hline
\end{tabular}

where $g_{\text {env }}$ and $T_{\text {env }}$ are gravity at the bottom of the atmosphere and mean atmospheric temperature, respectively. The quantity $R^{*}$ is the universal gas constant $\left(8.3144598 \mathrm{~J} \mathrm{~mol}^{-1} \mathrm{~K}^{-1}\right)$ and $\mu$ the mean molecular weight. The mass of the atmosphere $m_{\mathrm{env}}$ is directly related to the pressure $P_{\text {env }}$ as

$m_{\mathrm{env}}=4 \pi P_{\mathrm{env}} \frac{\left(R_{\mathrm{p}}-d_{\mathrm{env}}\right)^{2}}{g_{\mathrm{env}}}$,

where $R_{\mathrm{p}}-d_{\mathrm{env}}$ is the radius at the bottom of the atmosphere.

The atmosphere's constant temperature is defined as

$T_{\text {env }}=\alpha T_{\text {eff }} \sqrt{\frac{R_{\star}}{2 a}}$,

where $a$ is the semi-major axis. The factor $\alpha$ accounts for possible cooling and warming of the atmosphere and can vary between 0.5 and 1 , which is equivalent to the observed range of albedos among solar system bodies ( 0.05 for asteroids up to 0.96 for Eris). The upper limit of 1 is verified against the estimated $\alpha_{\max }$ (see Appendix A in Dorn et al. 2017), which takes possible greenhouse warming into account.

\subsubsection{Inference results}

Figure 5 summarises the interior estimates. Both planets have mantle compositions and core sizes that fit bulk density and the stellar abundance constraint. The core fraction of both planets is close to that of Venus and Earth $\left(\left(r_{\text {core }} / r_{\text {core }+ \text { mantle }}\right)_{\oplus}=0.53\right)$, which validates their denomination as super-Earths. Compared to planet $c$, the lower density of $10 \%$ of planet $b$ is associated with a slightly smaller core (by $10 \%$ ) and higher $f_{\text {mantle }}$ (by $45 \%$ ), which indicates that a significantly stronger reduction of mantle density is plausible given the data. The estimates of $f_{\text {mantle }}$ for planet $b$ and $c$ are $0.073_{-0.05}^{+0.06}$ and $0.05_{-0.04}^{+0.06}$, respectively. Factors of $f_{\text {mantle }}$ up to 0.25 can be associated with high melt fractions (for Earth-sized planets). Similar values can be achieved when the mantle composition is enriched by very refractory elements (i.e. $\mathrm{Al}, \mathrm{Ca}$ ).

It should be noted that differences between the interiors are small, since uncertainties on bulk densities are relatively large. The data allow for no difference in bulk densities. However, a significant (more than 5\%) difference exists with $70 \%$ probability. In this work, we used an interior model that allows us to quantify any possible difference in the rocky interiors of both planets. We assumed that any volatile layer is limited to a 100 bar atmosphere (similar to Venus) at maximum. Further arguments are necessary to evaluate whether a difference between the rocky interiors, specifically the mantle densities, can exist.
Nonetheless, because Bower et al. (2019) demonstrated that for Earth-sized planets a fully molten mantle is $25 \%$ less dense than a solidified mantle, this possibility must be considered, and it is interesting to investigate whether planet $b$ could be less dense because partially molten. Heating by irradiation from the host star would not be enough; the black-body equilibrium temperature for this planet is $1036 \mathrm{~K}$. Nevertheless, in the next subsection, we discuss a possible dynamical origin for the possible difference between HD $219134 b$ and $c$.

\subsection{Possible origin of a partial mantle melt for HD219134 b}

Large melt fractions may be sustained on planet $b$ by tidal heating. In the case of synchronous rotation with spin-orbit alignment, which is likely for close-in planets such as HD 219134 $b$, tidal dissipation acts only on planets on eccentric orbits around the star. The power is given by (see e.g. Lainey et al. 2009)

$\dot{E}=\frac{21}{2} \frac{k_{2}}{Q} \frac{\left(\omega R_{\mathrm{p}}\right)^{5}}{G} e^{2}$,

where $k_{2}$ is the Love number and $Q$ the quality factor of the planet of radius $R_{\mathrm{p}}$ and spin or orbital frequency $\omega$. The key parameter $\frac{k_{2}}{Q}$ depends on the internal properties of the body $^{8}$. The dissipated energy $\dot{E}$ heats the planet and damps the eccentricity of the orbit, ultimately leading to its circularisation and a reduction of the semi-major axis. To maintain tidal heating, the orbital eccentricity must be excited by the interaction with other secondary objects, as is the case for Jupiter's moon Io for instance. In order to investigate if tidal heating on planet $b$ is sufficient enough, we ran numerical simulations of the planetary system using the N-body code SyMBA (Duncan et al. 1998).

To build our initial conditions, we took the $e, \varpi$, orbital periods, $K$, and mid-transit time from Gillon et al. (2017). They measure a non-zero eccentricity for planets $c, f$, and $d$, but not for planet $b$, whose eccentricity is fixed to zero to fit the other orbital parameters. They do not provide data for the outermost two planets $g$ and $h$, but the long orbital periods of these planets make them unlikely to affect the inner four planets, and their orbital parameters suffer larger uncertainty so we neglect them in our simulations. We find that the eccentricity of planet $b$ is excited by the other planets. In absence of dissipation, the system is stable for at least $1 \mathrm{Gyr}$, and $e_{b}$ oscillates

8 For reference, it is of the order of $10^{-4,-5}$ for gas giant planets and about 0.025 for the Earth. 

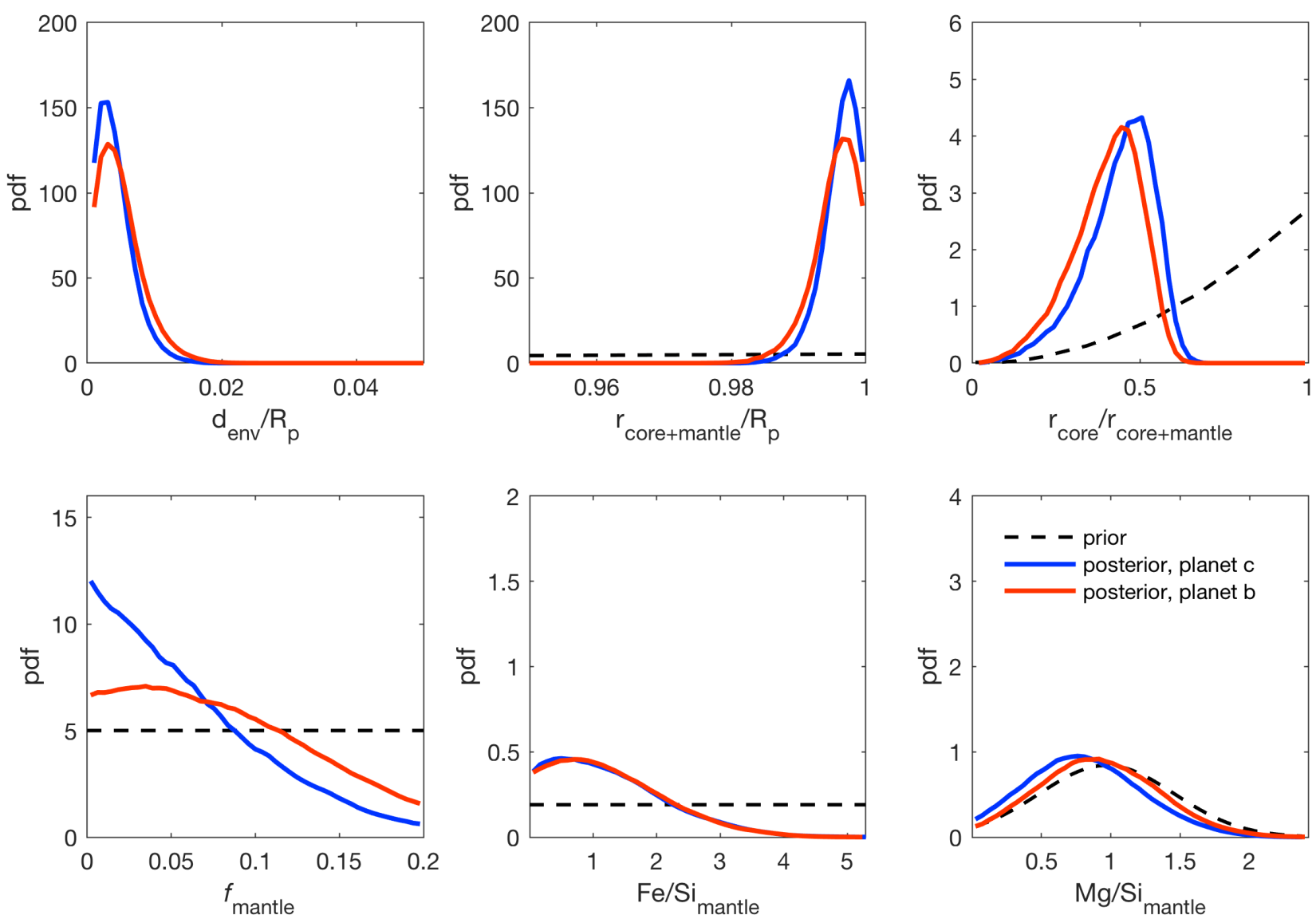

Fig. 5. One-dimensional marginalised posteriors of interior parameters: thickness of atmosphere $\left(d_{\text {env }}\right)$, size of rocky interior $\left(r_{\text {core }+ \text { mantle }}\right)$, core size $\left(r_{\text {core }}\right)$, fudge factor $f_{\text {mantle }}$, and mantle composition $\left(\mathrm{Fe} / \mathrm{Si}_{\text {mantle }}\right.$ and $\left.\mathrm{Mg} / \mathrm{Si}_{\text {mantle }}\right)$. The prior distribution is shown in dashed lines (except for $d_{\text {env }}$, for which no explicit prior is defined), while the posterior distribution is shown in solid lines for planets $b$ (red) and $c$ (blue).

freely between 0 and 0.13 with a period of a few thousand years ${ }^{9}$.

Introducing dissipation in planets $b$ and $c$, the eccentricities are damped and $e_{b}$ settles to a regime where it oscillates between 0.01 and 0.06 . The energy loss is balanced by an inward drift of the planets, mainly planet $b$. We note that the final value of the eccentricity is independent of the assumed value for $k_{2} / Q$, only the timescale of the evolution and inward drift are proportional to $k_{2} / Q$. Because of this dissipation, the period ratio $P_{c} / P_{b}$ increases with time, and it is possible that this ratio (which is now 2.19) was smaller than 2 , so that the $2: 1$ mean motion resonance was crossed recently. To check the effect of this phenomenon, we start planets $c$ and especially $b$ slightly out of their present position, inside the 2:1 mean motion resonance. Crossing the resonance at 14.6 Myrs kicks the eccentricities of planets $b$ and $c$, but this is quickly damped and the eccentricity of planet $b$ ends up oscillating between 0.005 and 0.037 with a period of $\sim 3000 \mathrm{yr}$ when it reaches its present semi-major axis at 73 Myrs, as shown in Fig. 6. Meanwhile, $e_{c}$ converges to 0.025 (while Gillon et al. 2017, find $0.062 \pm 0.039$ ). We checked that again,

\footnotetext{
9 Using initial circular orbits, i.e. assuming that the planets were fully formed locally in the protoplanetary disc, we observe no increase of the eccentricities of the four planets in 500 Myrs. This is not compatible with the observations of Gillon et al. (2017); this suggests that these four planets may not have acquired their final mass and/or orbits during the protoplanetary disc phase. A phase of giant impacts or the breaking of a resonance chain (Izidoro et al. 2017; Pichierri et al. 2018) could have happened in the early history of the system.
}

$k_{2} / Q$ has little influence on the final behaviour of the eccentricities, although the speed at which the resonance is crossed matters.

Using Eq. (11), $0.005<e_{b}<0.037$ gives a total power for the tidal heating oscillating in $5.6-308 \times 10^{16} \times\left(\frac{k_{2} / Q}{0.025}\right) \mathrm{W}$ for planet $b$ and around $2.1 \times 10^{16} \times\left(\frac{k_{2} / Q}{0.025}\right) \mathrm{W}$ for planet $c$. For reference, tidal heating in Io is of the order of $10^{14} \mathrm{~W}$ (Lainey et al. 2009) so that, assuming $k_{2} / Q=0.025$ like for Earth, planet $b$ receives at least 2 and up to 100 times more tidal heating per mass unit than Io (and almost 300 with $e_{b}=0.06$ ). In contrast, because in Eq. (11) the term $\left(\omega R_{p}\right)^{5}$ is 70 times smaller for planet $c$ than for planet $b$, all other parameters being equal, it should be heated much less. We find that it gets a bit less tidal heating than Io per mass unit, so it is unlikely to melt even partially. In the end, the idea of a partial (if not total) melt of the mantle of HD $219134 b$ to explain its possibly lower density than planet $c$ is strongly supported by dynamics. A refinement of the parameters of the system and a complete stability analysis would help but are beyond the scope of this paper.

\section{Summary and conclusions}

We present a new analysis of the exoplanetary system HD 219134. We observed the star with the VEGA/CHARA interferometer and measured an angular diameter of $1.035 \pm$ 0.021 mas and a radius of $0.726 \pm 0.014 R_{\odot}$. This radius is not significantly affected by the Gaia offset, but new values from the DR3 or DR4 will allow us to refine $R_{\star}$. We used the 

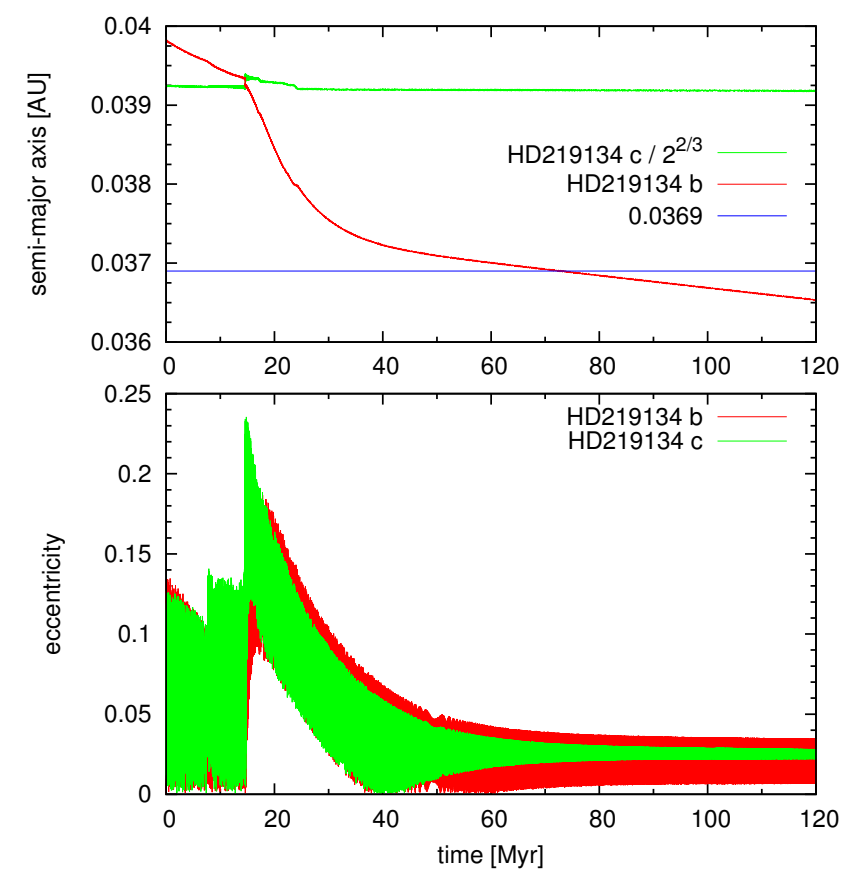

Fig. 6. Evolution of the inner two planets of the HD 219134 system under dissipation with $k_{2} / Q=0.025$ for both planets, and in presence of planets $f$ and $d$ of Gillon et al. (2017). Top panel: semi-major axis of planet $b$ (red curve), location of the 1:2 mean motion resonance with planet $c$ (green curve, that is $a_{c} / 2^{2 / 3}$ ), and present semi-major axis of planet $b$ (blue horizontal line). Bottom panel: eccentricities of planets $b$ (red) and $c$ (green).

transit parameters from Gillon et al. (2017) to measure the stellar density $\left(1.82 \pm 0.19 \rho_{\odot}\right)$ directly, and we directly derived from these two measurements the stellar mass $\left(0.696 \pm 0.078 M_{\odot}\right)$ and the correlation between $M_{\star}$ and $R_{\star}(0.46)$. We compare our parameters with those obtained with $\mathrm{C} 2 \mathrm{kSMO}$ and find that the range of masses is compatible with the directly measured mass, although the best model gives a mass $8 \%$ higher than the directly measured mass. This corresponds to an age of $9.3 \mathrm{Gyr}$, but a large range of ages is possible (0.2-9.3 Gyr). Similarly, previous indirect determinations of $M_{\star}$ show higher values than our measurement (see e.g. Boyajian et al. 2012b; Gillon et al. 2017), but it has to be noted that they are based on a larger $R_{\star}$.

The system includes two transiting exoplanets, HD $219134 b$ and $c$, for which we reassess the parameters. Using our new $R_{\star}$ and $M_{\star}$, we computed the PDF of the planetary masses and radii, which we find lower than previous estimates (since previous stellar parameters were higher), and the correlations between $M_{\mathrm{p}}$ and $R_{\mathrm{p}}$. These new values clearly validate the super-Earth nature of the two planets by putting them out of the gap in the exoplanetary radii distribution noticed by Fulton et al. (2017). We could thus derive the densities of the planets, which appear to differ by $10 \%$, although these values are possibly identical within the error bars (70\% chance that the difference is more than 5\%). More interestingly, planet $b$ has a lower density than planet $c$ despite its higher mass. Using Dorn et al. (2017) inference scheme, we show that this difference in density can be attributed to a slightly smaller core and/or a significantly lower mantle density. The latter might be due to a molten fraction. Tidal heating might be the cause of such a melting, as we investigated using the SyMBA N-body code. Excited by the other planets, the eccentricity of planets $b$ and $c$ reaches $\sim 0.02$ with tidal dissipation. This could lead to considerable heating for planet $b$ (100 times more than on Io per mass unit, possibly leading to partial melting of the mantle), while planet $c$ is too far from the star for tidal heating to be more intense than on Io. Hence, despite their possible density difference, planets $b$ and $c$ may have the same composition, as expected in all standard planet formation models.

The system of HD 219134 constitutes a benchmark case for both stellar and planetary sciences. Our direct estimation of the stellar radius and mass directly impacts the planetary parameters. Although within the error bars of the mass coming from $\mathrm{C} 2 \mathrm{kSMO}$, our new mass changes the planetary mass and the possibilities of interior structures compared to the possible solutions using the stellar models. Improving the precision of the transit light curves of the two planets would allow us to reduce the uncertainty on the stellar density, hence on the stellar mass. It would reduce the uncertainty on the planetary parameters even more, potentially answering the question of the density ratio of the two transiting super-Earths. More generally, measuring the stellar radius and density as we have done in this work is the most direct method to infer stellar (hence planetary) parameters and should be more extensively used; this approach will certainly be possible within the Transiting Exoplanet Survey Satellite (TESS) and PLAnetary Transits and Oscillations of stars (PLATO) missions era.

Acknowledgements. We thank the anonymous referee who brought useful comments and significantly helped improve our manuscript. R.L. has received funding from the European Union's Horizon 2020 research and innovation programme under the Marie Skłodowska-Curie grant agreement n. 664931. C.D. acknowledges support from the Swiss National Science Foundation under grant PZ00P2_174028. We thank A. Nakajima for her help with the initial conditions of the N-body simulations. This work is based upon observations obtained with the Georgia State University Center for High Angular Resolution Astronomy Array at Mount Wilson Observatory. The CHARA Array is supported by the National Science Foundation under Grants No. AST-1211929 and AST-1411654. This research has made use of the SIMBAD database, operated at CDS, Strasbourg, France. This research has made use of the Jean-Marie Mariotti Center SearchCal service ${ }^{10}$ co-developed by LAGRANGE and IPAG, and of CDS Astronomical Databases SIMBAD and VIZIER ${ }^{11}$. This work has made use of data from the European Space Agency (ESA) mission Gaia (https://www. cosmos.esa.int/gaia), processed by the Gaia Data Processing and Analysis Consortium (DPAC, https://www. cosmos.esa.int/web/gaia/dpac/ consortium). Funding for the DPAC has been provided by national institutions, in particular the institutions participating in the Gaia Multilateral Agreement.

\section{References}

Allende Prieto, C., Barklem, P. S., Lambert, D. L., \& Cunha, K. 2004, A\&A, 420, 183

Asplund, M., Grevesse, N., Sauval, A. J., \& Scott, P. 2009, ARA\&A, 47, 481

Baglin, A. 2003, Adv. Space Res., 31, 345

Baines, E. K., Döllinger, M. P., Cusano, F., et al. 2010, ApJ, 710, 1365

Boeche, C., \& Grebel, E. K. 2016, A\&A, 587, A2

Böhm-Vitense, E. 1958, Z. Astrophys., 46, 108

Bolis, R., Morard, G., Vinci, T., et al. 2016, Geophys. Res. Lett., 43, 9475

Bonneau, D., Clausse, J.-M., Delfosse, X., et al. 2006, A\&A, 456, 789

Boro Saikia, S., Marvin, C. J., Jeffers, S. V., et al. 2018, A\&A, 616, A108

Borucki, W. J., Koch, D., Basri, G., et al. 2010, Science, 327, 977

Bouchet, J., Mazevet, S., Morard, G., Guyot, F., \& Musella, R. 2013, Phys. Rev. B, 87, 094102

Bourgés, L., Lafrasse, S., Mella, G., et al. 2014, ASP Conf. Ser., 485, 223

Bourrier, V., Dumusque, X., Dorn, C., et al. 2018, A\&A, 619, A1

Bower, D. J., Kitzmann, D., Wolf, A. S., et al. 2019, A\&A, in press

http://doi.org/10.1051/0004-6361/201935710

Boyajian, T. S., McAlister, H. A., van Belle, G., et al. 2012a, ApJ, 746, 101

Boyajian, T. S., von Braun, K., van Belle, G., et al. 2012b, ApJ, 757, 112

Canuto, V. M., Goldman, I., \& Mazzitelli, I. 1996, ApJ, 473, 550

Casagrande, L., Flynn, C., Portinari, L., Girardi, L., \& Jimenez, R. 2007, MNRAS, 382, 1516

Chelli, A., Duvert, G., Bourgès, L., et al. 2016, A\&A, 589, A112

10 Available at http://WWW . jmmc . fr/searchcal

11 Available at http://cdsweb.u-strasbg.fr/ 
Claret, A., \& Bloemen, S. 2011, A\&A, 529, A75

Coelho, H. R., Chaplin, W. J., Basu, S., et al. 2015, MNRAS, 451, 3011

Connolly, J. 2009, Geochem. Geophys. Geosyst., 10, Q10014

Creevey, O. L., Thévenin, F., Boyajian, T. S., et al. 2012, A\&A, 545, A17

Creevey, O. L., Thévenin, F., Berio, P., et al. 2015, A\&A, 575, A26

Crida, A., Ligi, R., Dorn, C., \& Lebreton, Y. 2018a, ApJ, 860, 122

Crida, A., Ligi, R., Dorn, C., Borsa, F., \& Lebreton, Y. 2018b, Res. Notes Am. Astron. Soc., 2, 172

da Silva, R., Milone, A. d. C., \& Rocha-Pinto, H. J. 2015, A\&A, 580, A24

Dorn, C., \& Heng, K. 2018, ApJ, 853, 64

Dorn, C., Khan, A., Heng, K., et al. 2015, A\&A, 577, A83

Dorn, C., Venturini, J., Khan, A., et al. 2017, A\&A, 597, A37

Dorn, C., Harrison, J. H., Bonsor, A., \& Hands, T. O. 2018, MNRAS, 484, 712

Duncan, M. J., Levison, H. F., \& Lee, M. H. 1998, AJ, 116, 2067

Folsom, C. P., Fossati, L., Wood, B. E., et al. 2018, MNRAS, 481, 5286

Fortney, J. J., Marley, M. S., \& Barnes, J. W. 2007, ApJ, 659, 1661

Frasca, A., Covino, E., Spezzi, L., et al. 2009, A\&A, 508, 1313

Fulton, B. J., Petigura, E. A., Howard, A. W., et al. 2017, AJ, 154, 109

Gaia Collaboration (Prusti, T., et al.) 2016, A\&A, 595, A1

Gaia Collaboration (Brown, A. G. A., et al.) 2018, A\&A, 616, A1

Gennaro, M., Prada Moroni, P. G., \& Degl'Innocenti, S. 2010, A\&A, 518, A13

Gillon, M., Demory, B.-O., Van Grootel, V., et al. 2017, Nat. Astron., 1, 0056

Gray, R. O., Corbally, C. J., Garrison, R. F., McFadden, M. T., \& Robinson, P. E. 2003, AJ, 126, 2048

Grevesse, N., \& Noels, A. 1993, in Origin and Evolution of the Elements, eds. N. Prantzos, E. Vangioni-Flam, \& M. Casse (Singapore: World Scientific), 15 Gustafsson, B., Edvardsson, B., Eriksson, K., et al. 2008, A\&A, 486, 951

Hakim, K., Rivoldini, A., Van Hoolst, T., et al. 2018, Icarus, 313, 61

Heiter, U., \& Luck, R. E. 2003, AJ, 126, 2015

Henry, T. J., \& McCarthy, Jr., D. W. 1993, AJ, 106, 773

Hidalgo, S. L., Pietrinferni, A., Cassisi, S., et al. 2018, ApJ, 856, 125

Hinkel, N. R., Timmes, F., Young, P. A., Pagano, M. D., \& Turnbull, M. C. 2014, AJ, 148, 54

Huber, D., Ireland, M. J., Bedding, T. R., et al. 2012, ApJ, 760, 32

Izidoro, A., Ogihara, M., Raymond, S. N., et al. 2017, MNRAS, 470, 1750

Johnson, M. C., Endl, M., Cochran, W. D., et al. 2016, ApJ, 821, 74

Jones, B. W., Sleep, P. N., \& Underwood, D. R. 2006, ApJ, 649, 1010

Lainey, V., Arlot, J.-E., Karatekin, Ö., \& van Hoolst, T. 2009, Nature, 459, 957

Lallement, R., Vergely, J.-L., Valette, B., et al. 2014, A\&A, 561, A91

Lebreton, Y., \& Goupil, M. J. 2014, A\&A, 569, A21

Lebreton, Y., Goupil, M. J., \& Montalbán, J. 2014, EAS Pub. Ser., 65, 99

Lee, Y. S., Beers, T. C., Allende Prieto, C., et al. 2011, AJ, 141, 90

Lejeune, T., Cuisinier, F., \& Buser, R. 1997, VizieR Online Data Catalog: $\mathrm{J} / \mathrm{A}+\mathrm{A} / 412 / 50229$

Ligi, R., Mourard, D., Lagrange, A. M., et al. 2012, A\&A, 545, A5

Ligi, R., Mourard, D., Nardetto, N., \& Clausse, J.-M. 2013, J. Astron. Instrum., 02, 1340003

Ligi, R., Creevey, O., Mourard, D., et al. 2016, A\&A, 586, A94

Lindegren, L., Hernández, J., Bombrun, A., et al. 2018, A\&A, 616, A2

Luck, R. E., \& Heiter, U. 2005, AJ, 129, 1063

Luck, R. E., \& Heiter, U. 2006, AJ, 131, 3069

Luri, X., Brown, A. G. A., Sarro, L. M., et al. 2018, A\&A, 616, A9

Maldonado, J., Eiroa, C., Villaver, E., Montesinos, B., \& Mora, A. 2012, A\&A, 541, A40

Maldonado, J., Eiroa, C., Villaver, E., Montesinos, B., \& Mora, A. 2015, A\&A, 579, A20

Mamajek, E. E., \& Hillenbrand, L. A. 2008, ApJ, 687, 1264

Mishenina, T. V., Soubiran, C., Kovtyukh, V. V., \& Korotin, S. A. 2004, A\&A, 418, 551

Mishenina, T. V., Soubiran, C., Kovtyukh, V. V., Katsova, M. M., \& Livshits, M. A. 2012, A\&A, 547, A106

Mishenina, T. V., Pignatari, M., Korotin, S. A., et al. 2013, A\&A, 552, A128

Morel, P., \& Lebreton, Y. 2008, Ap\&SS, 316, 61

Motalebi, F., Udry, S., Gillon, M., et al. 2015, A\&A, 584, A72

Mourard, D., Clausse, J. M., Marcotto, A., et al. 2009, A\&A, 508, 1073
Mourard, D., Bério, P., Perraut, K., et al. 2011, A\&A, 531, A110

Mourard, D., Challouf, M., Ligi, R., et al. 2012, SPIE Conf. Ser., 8445, 84450K

Mourard, D., Monnier, J. D., Meilland, A., et al. 2015, A\&A, 577, A51

Nordlund, A., Stein, R. F., \& Asplund, M. 2009, Liv. Rev. Sol. Phys., 6, 2

Oja, T. 1993, A\&AS, 100, 591

Pichierri, G., Morbidelli, A., \& Crida, A. 2018, Celest. Mech. Dyn. Astron., 130, 54

Prugniel, P., Vauglin, I., \& Koleva, M. 2011, A\&A, 531, A165

Ramírez, I., Allende Prieto, C., \& Lambert, D. L. 2007, A\&A, 465, 271

Ramírez, I., Fish, J. R., Lambert, D. L., \& Allende Prieto, C. 2012, ApJ, 756, 46

Ramírez, I., Allende Prieto, C., \& Lambert, D. L. 2013, ApJ, 764, 78

Seager, S., \& Mallén-Ornelas, G. 2003, ApJ, 585, 1038

Silva Aguirre, V., Lund, M. N., Antia, H. M., et al. 2017, ApJ, 835, 173

Soubiran, C., Bienaymé, O., Mishenina, T. V., \& Kovtyukh, V. V. 2008, A\&A, 480, 91

Spaulding, D., McWilliams, R., Jeanloz, R., et al. 2012, Phys. Rev. Lett., 108, 065701

Stassun, K. G., \& Torres, G. 2018, ApJ, 862, 61

Stixrude, L., \& Lithgow-Bertelloni, C. 2011, Geophys. J. Int., 184, 1180

Tallon-Bosc, I., Tallon, M., Thiébaut, E., et al. 2008, SPIE Conf. Ser., 7013, 44

ten Brummelaar, T. A., McAlister, H. A., Ridgway, S. T., et al. 2005, ApJ, 628, 453

Valenti, J. A., \& Fischer, D. A. 2005, ApJS, 159, 141

Vogt, S. S., Burt, J., Meschiari, S., et al. 2015, ApJ, 814, 12

Wenger, M., Ochsenbein, F., Egret, D., et al. 2000, A\&AS, 143, 9

Wolf, A. S., \& Bower, D. J. 2018, Phys. Earth Planet. Inter., 278, 59

\section{Appendix A: Selected $\log (g)$ and metallicity from literature}

Table A.1. Parameters used to derive the $\log (g)$ and $[\mathrm{Fe} / \mathrm{H}]$ of Table 3 .

\begin{tabular}{lccl}
\hline \hline$T_{\text {eff }[\mathrm{K}]}$ & $\log (g)[\mathrm{dex}]$ & {$[\mathrm{Fe} / \mathrm{H}]$} & Reference \\
\hline 5100 & 4.40 & 0.10 & Heiter \& Luck (2003) \\
5100 & 4.65 & 0.04 & Luck \& Heiter (2006) \\
5100 & 4.65 & 0.04 & Luck \& Heiter (2005) \\
4798 & 4.55 & - & Gray et al. (2003) \\
4732 & 4.37 & 0.09 & Boeche \& Grebel (2016) \\
4858 & 4.67 & - & Maldonado et al. (2015) \\
5044 & 4.58 & 0.04 & da Silva et al. (2015) \\
4900 & 4.20 & 0.05 & Mishenina et al. (2013) \\
4833 & 4.59 & 0.00 & Ramírez et al. (2013) \\
4889 & 4.60 & 0.10 & Mishenina et al. (2012) \\
4833 & 4.59 & 0.00 & Ramírez et al. (2012) \\
- & - & 0.10 & Maldonado et al. (2012) \\
4851 & 4.37 & 0.07 & Lee et al. (2011) \\
4715 & 4.57 & 0.06 & Prugniel et al. (2011) \\
4710 & 4.50 & 0.20 & Frasca et al. (2009) \\
4913 & 4.51 & 0.08 & Soubiran et al. (2008) \\
4825 & 4.62 & 0.05 & Ramírez et al. (2007) \\
4835 & 4.56 & 0.12 & Valenti \& Fischer (2005) \\
4900 & 4.20 & 0.05 & Mishenina et al. (2004) \\
4743 & 4.63 & 0.12 & Allende Prieto et al. (2004) \\
\hline
\end{tabular}

Notes. We took into account the values given in the CDS database, removing those which were redundant and obtained before 2000 . 